\title{
Direct detection of tick-borne pathogens in Ixodes ricinus ticks collected from dogs and cats in Tuscany, Italy
}

\section{Valentina Chisu ( $\nabla$ valentinachisu@virgilio.it )}

Istituto Zooprofilattico Sperimentale della Sardegna G Pegreffi

Cipriano Foxi

Istituto Zooprofilattico Sperimentale della Sardegna G Pegreffi

\section{Gabriella Masu}

Istituto Zooprofilattico Sperimentale della Sardegna G Pegreffi

Barbara D' Amaddio

Istituto Zooprofilattico Sperimentale della Sardegna G Pegreffi

Giovanna Masala

Istituto Zooprofilattico Sperimentale della Sardegna G Pegreffi

Research article

Keywords: Ticks; tick-borne pathogens; Ixodes ricinus; Rickettsia spp.; Chlamydia spp.; Bartonella spp.

Posted Date: December 15th, 2019

DOI: https://doi.org/10.21203/rs.2.14517/v2

License: (c) (i) This work is licensed under a Creative Commons Attribution 4.0 International License. Read Full License 
The authors have withdrawn this preprint from Research Square 